\title{
On the core of a unicyclic graph
}

\author{
Vadim E. Levit \\ Ariel University Center of Samaria, Israel \\ Eugen Mandrescu \\ Holon Institute of Technology, Israel
}

Received 23 February 2011, accepted 22 December 2011, published online 10 April 2012

\begin{abstract}
A set $S \subseteq V$ is independent in a graph $G=(V, E)$ if no two vertices from $S$ are adjacent. By core $(G)$ we mean the intersection of all maximum independent sets. The independence number $\alpha(G)$ is the cardinality of a maximum independent set, while $\mu(G)$ is the size of a maximum matching in $G$.

A connected graph having only one cycle, say $C$, is a unicyclic graph. In this paper we prove that if $G$ is a unicyclic graph of order $n$ and $n-1=\alpha(G)+\mu(G)$, then core $(G)$ coincides with the union of cores of all trees in $G-C$.
\end{abstract}

Keywords: Maximum independent set, core, matching, unicyclic graph, König-Egerváry graph.

Math. Subj. Class.: 05C69, 05C70

\section{Introduction}

Throughout this paper $G=(V, E)$ is a simple (i.e., a finite, undirected, loopless and without multiple edges) graph with vertex set $V=V(G)$ and edge set $E=E(G)$. If $X \subset V$, then $G[X]$ is the subgraph of $G$ spanned by $X$. By $G-W$ we mean the subgraph $G[V-W]$, if $W \subset V(G)$. For $F \subset E(G)$, by $G-F$ we denote the partial subgraph of $G$ obtained by deleting the edges of $F$, and we use $G-e$, if $W=\{e\}$. If $A, B \subset V$ and $A \cap B=\emptyset$, then $(A, B)$ stands for the set $\{e=a b: a \in A, b \in B, e \in E\}$. The neighborhood of a vertex $v \in V$ is the set $N(v)=\{w: w \in V$ and $v w \in E\}$, and $N(A)=\cup\{N(v): v \in A\}, N[A]=A \cup N(A)$ for $A \subset V$. By $C_{n}, K_{n}$ we mean the chordless cycle on $n \geq 4$ vertices, and respectively the complete graph on $n \geq 1$ vertices.

A set $S$ of vertices is independent if no two vertices from $S$ are adjacent, and an independent set of maximum size will be referred to as a maximum independent set. The

E-mail addresses: levitv@ariel.ac.il (Vadim E. Levit), eugen_m@hit.ac.il (Eugen Mandrescu) 
independence number of $G$, denoted by $\alpha(G)$, is the size of a maximum independent set of $G$. Let $\Omega(G)$ denote the family $\{S: S$ is a maximum independent set of $G\}$, while

$$
\operatorname{core}(G)=\cap\{S: S \in \Omega(G)\}[11] .
$$

An edge $e \in E(G)$ is $\alpha$-critical whenever $\alpha(G-e)>\alpha(G)$. Notice that the inequalities $\alpha(G) \leq \alpha(G-e) \leq \alpha(G)+1$ hold for each edge $e$.

A matching (i.e., a set of non-incident edges of $G$ ) of maximum cardinality $\mu(G)$ is a maximum matching, and a perfect matching is one covering all vertices of $G$. An edge $e \in E(G)$ is $\mu$-critical provided $\mu(G-e)<\mu(G)$.

Theorem 1.1. [13] For every graph $G$ no $\alpha$-critical edge has an endpoint in $N[\operatorname{core}(G)]$.

It is well-known that

$$
\lfloor n / 2\rfloor+1 \leq \alpha(G)+\mu(G) \leq n
$$

hold for every graph $G$ with $n$ vertices. If $\alpha(G)+\mu(G)=n$, then $G$ is called a KönigEgerváry graph [3, 19]. Several properties of König-Egerváry graphs are presented in $[6,9,10,12,15,16]$.

It is known that every bipartite graph is a König-Egerváry graph as well [5, 8]. This class includes also non-bipartite graphs (see, for instance, the graph $G$ in Figure 1).

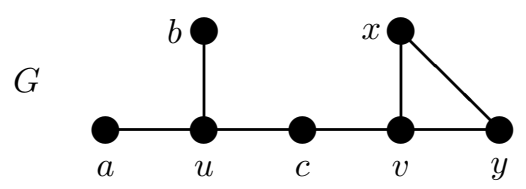

Figure 1: A König-Egerváry graph with $\alpha(G)=|\{a, b, c, x\}|$ and $\mu(G)=|\{a u, c v, x y\}|$.

Theorem 1.2. If $G$ is a König-Egerváry graph, then

(i) [12] every maximum matching matches $N(\operatorname{core}(G))$ into core $(G)$;

(ii) [13] $H=G-N[\operatorname{core}(G)]$ is a König-Egerváry graph with a perfect matching and each maximum matching of $H$ can be enlarged to a maximum matching of $G$.

The graph $G$ is called unicyclic if it is connected and has a unique cycle, which we denote by $C=(V(C), E(C))$. Let

$$
N_{1}(C)=\{v: v \in V(G)-V(C), N(v) \cap V(C) \neq \emptyset\},
$$

and $T_{x}=\left(V_{x}, E_{x}\right)$ be the tree of $G-x y$ containing $x$, where $x \in N_{1}(C), y \in V(C)$.
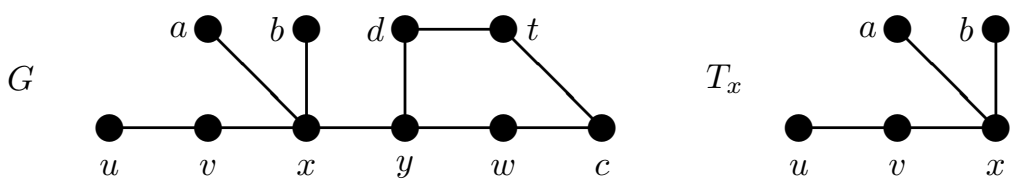

Figure 2: $G$ is a unicyclic non-König-Egerváry graph with $V(C)=\{y, d, t, c, w\}$.

Unicyclic graphs keep enjoying plenty of interest, as one can see, for instance, in [1, 4, 7, 14, 18, 20, 21].

In this paper we analyze the structure of $\operatorname{core}(G)$ for a unicyclic graph $G$. 


\section{Results}

If $G$ is a unicyclic graph, then there is an edge $e \in E(C)$, such that $\mu(G-e)=\mu(G)$, because for each pair of edges, consecutive on $C$, at most one could be $\mu$-critical. Let us mention that $\alpha(G) \leq \alpha(G-e) \leq \alpha(G)+1$ holds for each edge $e \in E(G)$. Every edge of the unique cycle could be $\alpha$-critical; e.g., the graph $G$ from Figure 2, which has also additional $\alpha$-critical edges (e.g., the edge $u v$ ).

Notice that the bipartite graph $T_{x}$ from Figure 2 has only two maximum matchings, namely, $M_{1}=\{a x, u v\}$ and $M_{2}=\{b x, u v\}$, while for each maximum matching there is a vertex in $\operatorname{core}\left(T_{x}\right)=\{a, b\}$ not saturated by that matching.

Lemma 2.1. For every bipartite graph $G$, a vertex $v \in \operatorname{core}(G)$ if and only if there exists a maximum matching that does not saturate $v$.

Proof. Since $v \in \operatorname{core}(G)$, it follows that $\alpha(G-v)=\alpha(G)-1$. Consequently, we have

$$
\alpha(G)+\mu(G)-1=|V(G)|-1=|V(G-v)|=\alpha(G-v)+\mu(G-v)
$$

which implies that $\mu(G)=\mu(G-v)$. In other words, there is a maximum matching in $G$ not saturating $v$.

Conversely, suppose that there exists a maximum matching in $G$ that does not saturate $v$. Since, by Theorem $1.2(i), N(\operatorname{core}(G))$ is matched into core $(G)$ by every maximum matching, it follows that $v \notin N(\operatorname{core}(G))$.

Assume that $v \notin \operatorname{core}(G)$. By Theorem 1.2(ii), every maximum matching $M$ of $G$ is of the form $M=M_{1} \cup M_{2}$, where $M_{1}$ matches $N(\operatorname{core}(G))$ into core $(G)$, while $M_{2}$ is a perfect matching of $G-N[\operatorname{core}(G)]$. Thus $v$ is saturated by every maximum matching of $G$, in contradiction with the hypothesis on $v$.

Remark 2.2. Lemma 2.1 fails for non-bipartite König-Egerváry graphs; e.g., every maximum matching of the graph $G$ from Figure 1 saturates $c \in \operatorname{core}(G)=\{a, b, c\}$.

Lemma 2.3. If $G$ is a unicyclic graph of order $n$, then $n-1 \leq \alpha(G)+\mu(G) \leq n$.

Proof. If $e=x y \in E(C)$, then $G-e$ is a tree, because $G$ is connected. Hence, $\alpha(G-e)+$ $\mu(G-e)=n$. Clearly, $\alpha(G-e) \leq \alpha(G)+1$, while $\mu(G-e) \leq \mu(G)$. Consequently, we get that

$$
n=\alpha(G-e)+\mu(G-e) \leq \alpha(G)+\mu(G)+1,
$$

which leads to $n-1 \leq \alpha(G)+\mu(G)$. The inequality $\alpha(G)+\mu(G) \leq n$ is true for every graph $G$.

Remark 2.4. If $G$ has $n$ vertices, $p$ connected components, say $H_{i}, 1 \leq i \leq p$, and each component contains only one cycle, then one can easily see that $n-p \leq \alpha(G)+\mu(G) \leq n$, because $\alpha(G)=\sum_{i=1}^{p} \alpha\left(H_{i}\right)$ and $\mu(G)=\sum_{i=1}^{p} \mu\left(H_{i}\right)$.

While $C_{2 k}, k \geq 2$, has no $\alpha$-critical edge at all, each edge of every odd cycle $C_{2 k-1}$, $k \geq 2$, is $\alpha$-critical. This property is partially inherited by unicyclic graphs.

Lemma 2.5. Let $G$ be a unicyclic graph of order $n$. Then $n-1=\alpha(G)+\mu(G)$ if and only if each edge of its unique cycle is $\alpha$-critical. 
Proof. Assume that $n-1=\alpha(G)+\mu(G)$. Since $G$ is connected, for each $e \in E(C)$ the graph $G-e$ is a tree. Hence, we have

$$
\alpha(G-e)-\alpha(G)+\mu(G-e)-\mu(G)=1
$$

which implies $\mu(G-e)=\mu(G)$ and $\alpha(G-e)=\alpha(G)+1$, since

$$
-1 \leq \mu(G-e)-\mu(G) \leq 0 \leq \alpha(G-e)-\alpha(G) \leq 1 .
$$

In other words, every $e \in E(C)$ is $\alpha$-critical.

Conversely, let $e \in E(C)$ be such that $\mu(G-e)=\mu(G)$; such an edge exists, because no two consecutive edges on $C$ could be $\mu$-critical. Since $e$ is $\alpha$-critical, and $G-e$ is a tree, we infer that

$$
n-1=\alpha(G-e)+\mu(G-e)-1=\alpha(G)+\mu(G),
$$

and this completes the proof.

Combining Lemma 2.5 and Theorem 1.1, we infer the following.

Corollary 2.6. If $G$ is a unicyclic non-König-Egerváry graph, then no vertex of its unique cycle belongs to $N[\operatorname{core}(G)]$.

Remark 2.7. Corollary 2.6 is true also for some unicyclic König-Egerváry graphs; e.g., the graph $H_{1}$ from Figure 3. However, the König-Egerváry graph $H_{2}$ from the same figure satisfies $N\left[\operatorname{core}\left(H_{2}\right)\right] \cap V(C)=\{u\} \neq \emptyset$.
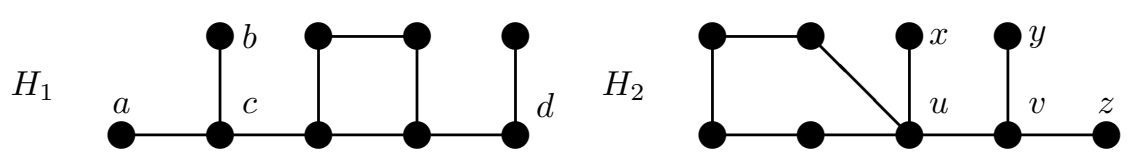

Figure 3: $H_{1}$ and $H_{2}$ have $N\left[\operatorname{core}\left(H_{1}\right)\right]=\{a, b, c\}, N\left[\operatorname{core}\left(H_{2}\right)\right]=\{x, y, z, u, v\}$.

Lemma 2.8. Let $G$ be a unicyclic graph of order $n$. If there exists some $x \in N_{1}(C)$, such that $x \in \operatorname{core}\left(T_{x}\right)$, then $G$ is a König-Egerváry graph.

Proof. Let $x \in \operatorname{core}\left(T_{x}\right), y \in N(x) \cap V(C)$, and $z \in N(y) \cap V(C)$. Suppose, to the contrary, that $G$ is not a König-Egerváry graph. By Lemmas 2.3 and 2.5, the edge $y z$ is $\alpha$-critical. Hence $y \notin \operatorname{core}(G)$, which implies that $\alpha(G)=\alpha(G-y)$. In accordance with Lemma 2.1, there exists a maximum matching $M_{x}$ of $T_{x}$ not saturating $x$. Combining $M_{x}$ with a maximum matching of $G-y-T_{x}$ we get a maximum matching $M_{y}$ of $G-y$. Hence $M_{y} \cup\{x y\}$ is a matching of $G$, which results in $\mu(G) \geq \mu(G-y)+1$. Therefore, using Lemma 2.3 and having in mind that $G-y$ is a forest of order $n-1$, we get the following contradiction

$$
n-1=\alpha(G)+\mu(G) \geq \alpha(G-y)+\mu(G-y)+1=n-1+1=n,
$$

that completes the proof. 
Remark 2.9. The converse of Lemma 2.8 is not generally true; e.g., the graph $H_{1}$ from Figure 3 is a unicyclic König-Egerváry graph, while both $c \notin \operatorname{core}\left(T_{c}\right)=\{a, b\}$, and $d \notin \operatorname{core}\left(T_{d}\right)=\emptyset$.

Theorem 2.10. If $G$ is a unicyclic non-König-Egerváry graph, then

$$
\text { core }(G)=\cup\left\{\operatorname{core}\left(T_{x}\right): x \in N_{1}(C)\right\} .
$$

Proof. Claim 1. Every maximum independent set of $T_{x}$ may be enlarged to some maximum independent set of $G$, for each $x \in N_{1}(C)$.

Let $A \in \Omega\left(T_{x}\right), y \in N(x) \cap V(C)$, and $z \in N(y) \cap V(C)$. According to Lemma 2.5, the edge $y z$ is $\alpha$-critical. Hence there exist $S_{y} \in \Omega(G), S_{y z} \in \Omega(G-y z)$, such that $y \in S_{y}$ and $y, z \in S_{y z}$.

Case 1. Assume that $x \notin A$.

If $\left|S_{y}-V\left(T_{x}\right)\right|<\alpha\left(G-T_{x}\right)=\left|S_{0}\right|$, where $S_{0} \in \Omega\left(G-T_{x}\right)$, then the set $S_{1}=$ $S_{0} \cup\left(S_{y} \cap V\left(T_{x}\right)\right)$ is independent in $G$, and we get the contradiction

$$
\alpha(G)=\left|S_{y}-V\left(T_{x}\right)\right|+\left|S_{y} \cap V\left(T_{x}\right)\right|<\left|S_{0}\right|+\left|S_{y} \cap V\left(T_{x}\right)\right|=\left|S_{1}\right| .
$$

Therefore, we have $\left|S_{y}-V\left(T_{x}\right)\right|=\alpha\left(G-T_{x}\right)$. Then $A \cup\left(S_{y}-V\left(T_{x}\right)\right) \in \Omega(G)$, otherwise we obtain the following contradiction

$$
\left|S_{y}-V\left(T_{x}\right)\right|+|A|<\alpha(G) \leq \alpha\left(G-T_{x}\right)+\alpha\left(T_{x}\right)=\left|S_{y}-V\left(T_{x}\right)\right|+|A| .
$$

Case 2. Assume now that $x \in A$.

Then we have $|A| \geq\left|S_{y z} \cap V\left(T_{x}\right)\right|$, because $S_{y z} \cap V\left(T_{x}\right)$ is independent in $T_{x}$. Hence we infer

$$
\begin{gathered}
\alpha(G)=\left|S_{y z}-\{y\}\right| \leq\left|\left(S_{y z}-\{y\}-\left(S_{y z} \cap V\left(T_{x}\right)\right)\right) \cup A\right|= \\
=\left|\left(S_{y z}-\{y\}-V\left(T_{x}\right)\right) \cup A\right| .
\end{gathered}
$$

Since $W=\left(S_{y z}-\{y\}-V\left(T_{x}\right)\right) \cup A$ is independent and its size is $\alpha(G)$ at least, it follows that $W$ is also a maximum independent set, i.e., we have $A \subseteq W \in \Omega(G)$, as needed.

Claim 2. $S \cap V\left(T_{x}\right) \in \Omega\left(T_{x}\right)$ for every $S \in \Omega(G)$ and each $x \in N_{1}(C)$.

Let $S \in \Omega(G)$, and suppose, to the contrary, that $A=S \cap V\left(T_{x}\right) \notin \Omega\left(T_{x}\right)$. By Lemma 2.8, $x \notin \operatorname{core}\left(T_{x}\right)$. Thus we can change $A$ for some $B \in \Omega\left(T_{x}\right)$ not containing $x$. The set $(S-A) \cup B$ is clearly independent in $G$, and this leads to the contradiction $|(S-A) \cup B|=|S-A|+|B|>|S|=\alpha(G)$.

Combining Claims 1 and 2, we infer that:

$$
\begin{aligned}
\operatorname{core}\left(T_{x}\right) & =\cap\left\{A: A \in \Omega\left(T_{x}\right)\right\}=\cap\left\{S \cap V\left(T_{x}\right): S \in \Omega(G)\right\} \\
& =(\cap\{S: S \in \Omega(G)\}) \cap V\left(T_{x}\right)=\operatorname{core}(G) \cap V\left(T_{x}\right),
\end{aligned}
$$

which clearly implies

$$
\operatorname{core}(G)=\cup\left\{\operatorname{core}\left(T_{x}\right): x \in N(V(C))-V(C)\right\}
$$

as required. 
Remark 2.11. The assertion in Theorem 2.10 may fail for:

(i) bipartite unicyclic graphs; for example, the graphs $H_{1}, H_{2}$ from Figure 4 satisfy

$$
\begin{aligned}
& \operatorname{core}\left(H_{1}\right)=\cup\left\{\operatorname{core}\left(T_{x}\right): x \in N_{1}(C)\right\}, \text { and } \\
& \text { core }\left(H_{2}\right) \neq\{x, z\}=\cup\left\{\operatorname{core}\left(T_{x}\right): x \in N_{1}(C)\right\} ;
\end{aligned}
$$
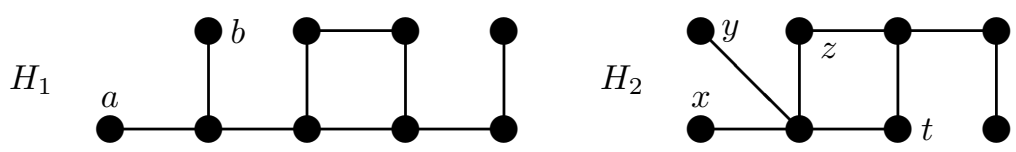

Figure 4: $H_{1}, H_{2}$ are bipartite unicyclic graphs, core $\left(H_{1}\right)=\{a, b\}, \operatorname{core}\left(H_{2}\right)=$ $\{t, x, y, z\}$.

(ii) non-bipartite König-Egerváry unicyclic graphs; for instance,

$$
\begin{aligned}
& \text { core }\left(G_{2}\right) \neq\{t, z\}=\cup\left\{\operatorname{core}\left(T_{x}\right): x \in N_{1}(C)\right\}, \text { while } \\
& \text { core }\left(G_{1}\right)=\cup\left\{\operatorname{core}\left(T_{x}\right): x \in N_{1}(C)\right\},
\end{aligned}
$$

where $G_{1}$ and $G_{2}$ are from Figure 5 .
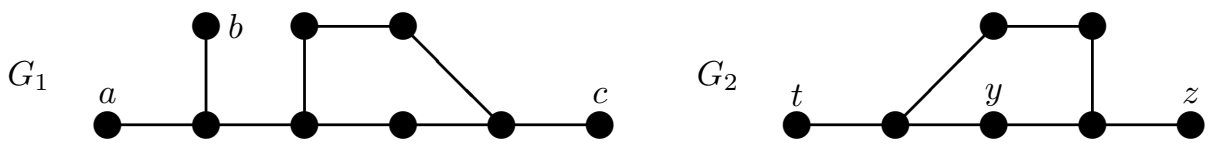

Figure 5: $G_{1}, G_{2}$ are König-Egerváry graphs, core $\left(G_{1}\right)=\{a, b, c\}, \operatorname{core}\left(G_{2}\right)=\{t, y, z\}$.

It is worth mentioning that the problem of whether there are vertices in a given graph $G$ belonging to core $(G)$ is NP-hard [2]. In [17] we have presented both sequential and parallel algorithms finding core $(G)$ in polynomial time for König-Egerváry graphs. By Theorem 2.10, a unicyclic graph is either a König-Egerváry graph or its core $(G)$ equals a union of cores of a finite number of some special subtrees. Therefore, we get the following.

Corollary 2.12. If $G$ is a unicyclic graph, then core $(G)$ is computable in polynomial time.

\section{Conclusions}

The main purpose of this paper is to investigate the structure of core $(G)$ for unicyclic graphs. One the one hand, we have succeeded to represent core $(G)$ as the union of cores of some specific subtrees of a non König-Egerváry unicyclic graph $G$. On the other hand, it is still not clear if there exists a characterization of this kind for bipartite unicyclic graphs and/or non-bipartite König-Egerváry graphs.

\section{References}

[1] F. Belardo, M. Li, M. Enzo, S. K. Simić and J. Wang, On the spectral radius of unicyclic graphs with prescribed degree sequence, Linear Algebra Appl. 432 (2010), 2323-2334. 
[2] E. Boros, M. C. Golumbic and V. E. Levit, On the number of vertices belonging to all maximum stable sets of a graph, Discrete Appl. Math. 124 (2002), 17-25.

[3] R. W. Deming, Independence numbers of graphs - an extension of the König-Egerváry theorem, Discrete Math. 27 (1979), 23-33.

[4] Z. Du, B. Zhou and N. Trinajstić, Minimum sum-connectivity indices of trees and unicyclic graphs of a given matching number, J. Math. Chem. 47 (2010), 842-855.

[5] E. Egerváry, On combinatorial properties of matrices, Matematikai Lapok 38 (1931), 16-28.

[6] F. Gavril, Testing for equality between maximum matching and minimum node covering, Inform. Process. Lett. 6 (1977), 199-202.

[7] B. Huo, S. Ji and X. Li, Note on unicyclic graphs with given number of pendent vertices and minimal energy, Linear Algebra Appl. 433 (2010), 1381-1387.

[8] D. König, Graphen und Matrizen, Matematikai Lapok 38 (1931), 116-119.

[9] E. Korach, T. Nguyen and B. Peis, Subgraph characterization of red/blue-split graphs and König-Egerváry graphs, Proceedings of the Seventeenth Annual ACM-SIAM Symposium on Discrete Algorithms, ACM Press, 2006, pp. 842-850.

[10] C. E. Larson, The critical independence number and an independence decomposition, Eur. $J$. Combin. 32 (2011), 294-300.

[11] V. E. Levit and E. Mandrescu, Combinatorial properties of the family of maximum stable sets of a graph, Discrete Appl. Math. 117 (2002), 149-161.

[12] V. E. Levit and E. Mandrescu, On $\alpha^{+}$-stable König-Egerváry graphs, Discrete Math. 263 (2003), 179-190.

[13] V. E. Levit and E. Mandrescu, On $\alpha$-critical edges in König-Egervary graphs, Discrete Math. 306 (2006), 1684-1693.

[14] V. E. Levit and E. Mandrescu, Greedoids on vertex sets of unicycle graphs, Congressus Numerantium 197 (2009), 183-191.

[15] V. E. Levit and E. Mandrescu, A characterization of König-Egerváry graphs using a common property of all maximum matchings, Electronic Notes in Discrete Math. 38 (2011), 565-570.

[16] V. E. Levit and E. Mandrescu, Critical independent sets and König-Egerváry graphs, Graph. Combinator. 28 (2012), 243-250.

[17] V. E. Levit and E. Mandrescu, An algorithm computing the core of a König-Egerváry graph, 2011, arXiv:1102.1141 [cs.DM], 8 pp.

[18] J. Li, J. Guo and W. C. Shiu, The smallest values of algebraic connectivity for unicyclic graphs, Discrete Appl. Math. 158 (2010), 1633-1643.

[19] F. Sterboul, A characterization of the graphs in which the transversal number equals the matching number, J. Comb. Theory B 27 (1979), 228-229.

[20] Y. Wu and J. Shu, The spread of the unicyclic graphs, Eur. J. Combin. 31 (2010), 411-418.

[21] M. Zhai, R. Liu and J. Shu, Minimizing the least eigenvalue of unicyclic graphs with fixed diameter, Discrete Math. 310 (2010), 947-955. 